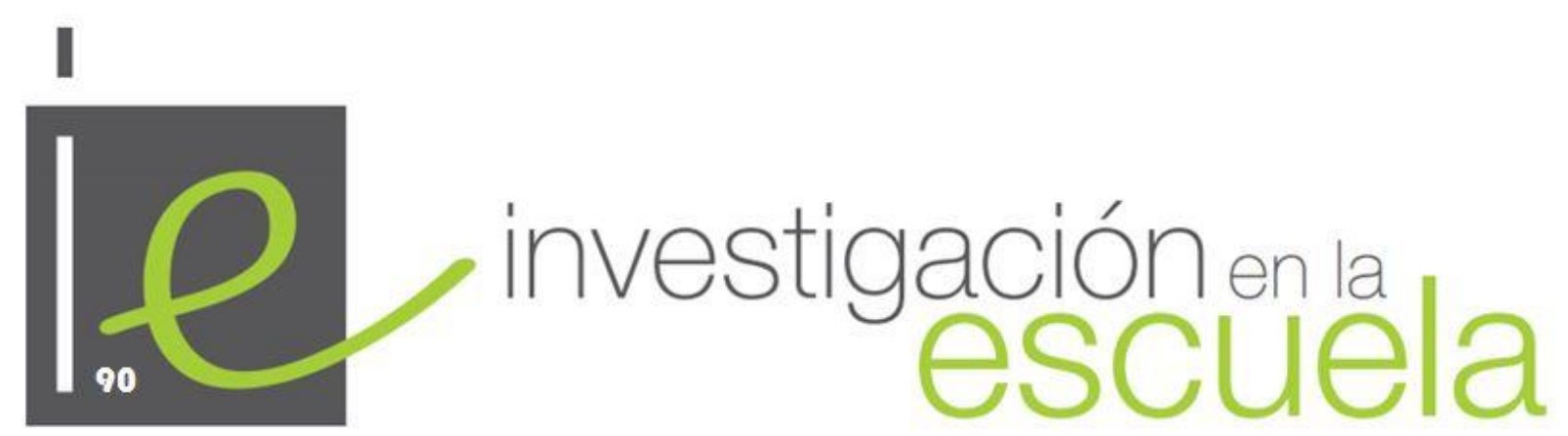

Revista académica evaluada por pares y de acceso abierto

\title{
Educando desde los sabores. Una experiencia antropoeducativa en una población de riesgo
}

\author{
Juan Carlos Romero Villadóniga \\ IES La Marisma \\ España
}

Citación: Romero, J. (2016). Educado desde los sabores. Una experiencia antropoeducativa en una población de riesgo. Investigación en la Escuela, 90, 34-48. Recuperado de http://www .investigacionenlae scuela.es/articulos/R90/R90-3.pdf

Resumen: En una sociedad globalizada, donde el conflicto se entiende como una mera transgresión y no como una oportunidad para la reflexión y el cambio, las poblaciones de adolescentes en riesgo de exclusión social cada vez ven más limitadas sus posibilidades de acceso a la comunidad. Es entonces cuando el conflicto se convierte en violencia, pues las herramientas formativas han fallado en su primera concepción, la socialización del individuo y su conversión en ciudadanos libres y responsables. Tan sólo asumiendo la complejidad del ser humano como tal, se puede llegar a una inserción plena, debiendo ponerse la institución educativa al servicio de sus necesidades y particulares, y nunca al contrario. Por esta razón, talleres motivacionales como los que se explican en el actual trabajo no son sino respuestas de atención a una diversidad que clama por una educación ajustada a sus necesidades.

Palabras clave: "Problemas sociales"; “integración racial”; “complejidad"; "capital cultural”.

Educating from flavour. an anthro-educational experience focused on a population group at risk.

Abstract: Within the context of a global society, the term 'conflict' is understood as a mere offence, rather than interpreted to mean an opportunity for critical reflection and social change. Thus, the 
segments of youth population at risk of poverty and social exclusion are increasingly constrained in their possibilities to gain access and participate in society at all levels. It is in these instances that the conflict conceived as an offence persists and evolves into violence, since training measures have failed to bring the desired results: the socialization of the individual as well as the expectations of becoming responsible citizens and free thinking human beings.

The complexity of the human nature constitutes an essential step towards full integration into society, bearing in mind that educational institutions should serve individuals and meet their needs; never the other way round. For this reason, the implementation of motivational workshops and programs, such as those explained more fully in the section below; provides an appropriate response to diversity in learning environments where individuals from different backgrounds claim their rights to teaching practices adapted to their needs.

Key words: "Social issues"; "racial integration"; "complexity"; "cultural capital".

\section{Eduquer des saveurs. Une expérience anthropologie dans une population à risque.}

Resumè: Dans une société mondialisée, où le conflit est entendu comme une simple transgression et non comme une opportunité pour la réflexion et le changement, les populations d'adolescents à risque d'exclusion sociale sont de plus en plus limité leur accès à la communauté. Voilà où le conflit devient violent, parce que les outils de formation ont échoué dans leur première conception, la socialisation des individus et les convertir en citoyens libres et responsables. Juste en supposant que la complexité de l'être humain en tant que tel, il peut atteindre une pleine intégration et devrait être l'établissement d'enseignement au service de vos besoins et des individus, et jamais l'inverse. Pour cette raison, ateliers de motivation, comme expliqué dans le travail en cours ne sont que des réponses de soins à une diversité qui crie pour l'éducation adaptée à leurs besoins.

Mosts clé: "Problèmes sociaux"; "l'intégration raciale"; "complexité"; "le capital culturel".

\section{Introducción}

En un mundo en continuo cambio, donde el espacio y el tiempo han experimentado una transformación sin límites (Augé, 2008), el triunfo de la sobremodernidad frente a formas más coherentes de existencia ha desembocado en una pérdida de referentes identitarios, y con ello una crisis de valores.

Dichos cambios afectan aún más, si cabe, a colectivos en riesgo de exclusión social, pues la brecha con respecto al resto se dilata hasta convertirse en un abismo. A nivel educativo, esta fisura se traduce en elevados porcentajes de abandono escolar a edades tempranas, $24,9 \%$ en el curso 2013-2014 (MECD, 2015), absentismo itinerante con la consiguiente pérdida de un seguimiento formativo continuo, escasa reinserción real de alumnado que ha abandonado el sistema educativo antes de finalizar su período de promoción, así como una pérdida generalizada de autoestima, lo que desemboca en la asunción de roles de resistencia.

Y qué decir sobre la realidad de los centros educativos con alumnado en riesgo de exclusión social. Proyectos pedagógicos poco ajustados a la realidad tanto del docente como del discente, profesorado incapaz de poder atender a una diversidad que se le escapa de sus manos y que logra actuar gracias a su denostado esfuerzo y compromiso unilateral, son realidades cotidianas, como bien apuntan tantos y tanto autores (Jociles \& Franzé, 2008 y 2011; García \& Olmos, 2012; Soriano, 2009 y 2011)

Por esta razón, se hace necesario un cambio de perspectiva, pararse a pensar sobre qué camino debe tomar el sistema para tratar el problema del alumnado en riesgo de exclusión. Dejar a 
un lado el programa para reformular todo el entramado simbólico (Geertz, 1987) que genera la acción educativa, y partir de la necesidad de formar "sujetos reflexivos", no ciudadanos uniformes sin capacidad crítica. La educación debe ser universal en sus principios, pero al mismo tiempo particular, atendiendo a la diversidad como una oportunidad única de generar espacios de conciencia (Del Toro, 2012) alejados de la actual macdonalización de la sociedad, que genera procesos y espacios de exclusión, especialmente en los colectivos más vulnerables.

\section{Una educación de la complejidad para contextos de riesgo}

Sin duda alguna, el análisis de población en riesgo de exclusión social ha sido un tema muy frecuentado en la literatura educativa (Subirats, 2004; Velaz de Medrano,2005).

Cambios normativos de forma desproporcionada y sin una actitud reflexiva, propuestas aleatorias de intervención con este tipo de colectivo que no se ajustan a las necesidades ni aspiraciones de éstos (Carabaña, 2004), hacen que el desfase existente entre el sistema y las necesidades reales de sus beneficiarios se haga cada vez más evidente. Ello provoca un déficit formativo que dificulta la posterior incorporación al mundo laboral y social (Bolívar y López, 2009), enquistando, como bucle recursivo, una situación que en teoría trataba de evitar el sistema educativo.

En una sociedad compleja, donde todos los procesos se encuentran conectados entre sí por medio de tramas ocultas (Capra, 2003), muchas de ellas imperceptibles pero de gran trascendencia, no se puede atender a la diversidad desde la simplicidad programática, so pena de generar inconscientemente espacios institucionalizados de segregación que potencian, paradójicamente, un carácter excluyente (Luengo, 2004).

De esta forma, el papel que juegan los actores educativos locales en el fenómeno de exclusión social son más que determinantes, pues dependiendo de las decisiones que se tomen en los diferentes niveles (familia, claustro, equipos directivos, equipos de zona), se estará generando un espacio segregador o bien justamente lo contrario. Por ello es necesario partir de una misma filosofía de trabajo, con la preeminencia del sujeto frente al proceso, con unos mismos fines y metodologías, estableciendo unas formas comunes de intervención dentro y fuera del aula, así como de gestión del conflicto que se suele producir de forma cotidiana.

Esta reflexión nos debe conducir a otra no menos importante, la importancia de "la capacidad de escucha activa del profesorado", algo que por desgracia no se practica con la asiduidad recomendada. En este sentido, el valor del profesor nunca es cuestionado en el actual sistema educativo, quedando siempre en un segundo plano las inquietudes y el mundo del adolescente. Unas veces de forma paternalista, otras impositiva, el profesor adoctrina y somete a juicio el imaginario del alumno, en un intento de configurar una identidad más próxima a lo que se pide en la sociedad actual. No obstante, como apunta Achilli (1996:630): “ el niño construye auto imágenes que influyen en los procesos de aprendizaje y en el desenvolvimiento de su vida escolar. Va configurando lo que hemos denominado identidades escolarizadas", de ahí la enorme responsabilidad del educador. Ello genera una desconexión entre lo que el alumno en riesgo de exclusión necesita y reclama, y lo que se le ofrece. La educación jamás debe partir de una concepción “Top Down” donde el conocimiento vaya permeando de forma espontánea, sin tener en cuenta a sus receptores, sino justamente lo contrario, debe orientarse a partir de una filosofía "Botton Up", donde el conocimiento sea reclamado desde las bases. En cierto modo, hablamos de una verdadera educación inclusiva que parte de las necesidades y realidades sociales del alumnado y no de las constricciones de un sistema educativo que se muestra como un dinosaurio normativo alejado de la realidad del aula.

Todas las medidas de atención a la diversidad que se quieran implementar para corregir estos déficits no sirven absolutamente de nada si no es partiendo de dicha premisa, ya que lo único que se 
está generando en la mayoría de los casos, es una persistencia de la desigualdad (Bolívar y López, 2009); eso sí, revestida quizás de un altruismo normativo sin límites, pero alejado de las necesidades reales. Sería lo que Malinowsky (1995) enunciaba como la disparidad entre la cultura irreal y la real. En este sentido resulta necesario encontrar puntos de encuentro entre las necesidades de unos y los ofrecimientos de otros. Adaptarse a las peculiares cosmogonías simbólicas del alumnado en riesgo de exclusión es atender a una verdadera diversidad de forma inclusiva, ya que se forma un vínculo entre el mundo docente y el discente.

Por esta razón, se hace necesario un cambio de rumbo, repensar la educación y potenciar los lazos ocultos de solidaridad presentes en los procesos, y eso no se puede hacer sino asumiendo la complejidad de la acción, es decir asumiendo la existencia de un tejido de constituyentes heterogéneos que se presentan indisolublemente unidos en una aparente paradoja de unicidad y múltiplicidad (Morin, 2004). Hay que abandonar definitivamente una concepción educativa en la que no tengan cabida elementos de la vida cotidiana como el azar, la incertidumbre, la indeterminación o el desorden, pues forman parte consustancial del ser humano y negarlos no hace sino abrir puertas al conflicto.

La intervención en contextos de vulnerabilidad necesita estar libre de preconcepciones y estereotipos que lastran la acción educativa. No debe priorizar el currículum por encima del valor del SUJETO, pues sólo a partir de la intervención individualizada se puede lograr una verdadera atención a la diversidad, asumiendo el azar como un elemento más de la acción.

Para ello, es conveniente reconducir la concepción humana a su verdadero nivel de complejidad, considerando todas las variables presentes en su configuración. Partir de la necesidad de asumir al sujeto como un ser en contante proceso dialógico y reflexivo que le empuja, de forma autónoma y libre, a una permanente reformulación de su conciencia, constreñida por unas características sociales, biológicas y ambientales que lastran su crecimiento personal y le impiden acceder a una mayor autonomía.

En esta concepción el sujeto se auto-observa en todo momento y es consciente de las paradojas que genera en el seno de su comunidad y con él mismo, permitiéndole conocer los "puntos ciegos" que no son empíricamente observables si no es por medio de este ejercicio. De nada sirven los estímulos externos en el individuo en riesgo de exclusión si durante todas las fases de la intervención no hay un acompañamiento de un cierto extrañamiento interior. La clave de todo el proceso va a venir de la mano de la reflexividad, definida como "aquella operación cognitiva en la que cada sujeto objetiviza dentro de su propia subjetividad, la realidad que lo circunda. Esta operación, a su vez, le incentiva la auto-observación y la autocrítica, aportando la auto-eco-reflexividad" (Pozzoli, 2006: 25).

En otras palabras, el objetivo que se quiere promover no es sino el descubrimiento interno de sí mismo, y a partir de ahí, generar nuevas formas de relacionarse con su entorno más próximo. Como sujeto en la complejidad, no puede ser separado del contexto de pertenencia al que está adscrito, pero sí puede generar, por medio de la reflexión, cambios que repercutan en el entorno. Partiendo de estas premisas filosóficas, y hasta incluso diría entrópicas con el actual sistema educativo, el proceso de investigación que estamos desarrollando parte de un primer objetivo fundamental, la necesidad de caracterizar al alumnado en contexto de riesgo desde una perspectiva compleja, analizando las intersecciones subyacentes no sólo en el hecho educativo, sino también en la configuración de los sujetos. Sólo así podremos tener una idea aproximada de la envergadura del problema. Por esta razón se recurre a la etnografía escolar, convirtiéndose en nuestra herramienta analítica cotidiana. Numerosos autores han entrado en el capítulo de su definición (Woods, 1987; Goetz y Lecompte, 1988; Martínez Rodríguez, 1990; Aguirre Baztán, 1995; Sanchís Ochoa y Cantón Delgado, 1995; Serra, 2004), coincidiendo en líneas generales en ser "un estudio descriptivo (graphos) de la cultura (ethos) de una comunidad” (Aguirre Baztán, 1995:3). 
Igualmente, nuestra investigación plantea, como segundo objetivo, la necesidad de conocer las concepciones del profesorado, así como las dinámicas que provocan el conflicto, a fin de determinar pautas y causalidades que sirvan como elementos de corrección en las relaciones profesor-alumnado. Por último, el tercer objetivo es la creación de un programa de mejora de la conflictividad en el aula mediante su auto-modificación conductual, a partir de una estrategia de trabajo colaborativo en un contexto diferente a los espacios de coacción habituales que se generan en el aula y que constituyen una de sus bases.

Con esa finalidad, se puso en marcha, primero de forma experimental, y luego asentándose en el tiempo (pues ya va por su sexta edición), un taller de cocina multicultural donde el alumnado es el principal recurso, tomando todas las decisiones y solucionando de forma asertiva los problemas que se plantean cotidianamente.

Gracias a la labor antropoeducativa desarrollada, no sólo se han detectado las causas que explican la conflictividad en alumnado en riesgo, sino que además se han establecido medidas correctoras igualmente mensuradas de forma estadística, en un ejercicio de prospectiva, dando utilidad a la investigación, así como a la posterior intervención.

\section{Conociendo los ingredientes. Metodología empleada}

El trabajo que aquí se plantea es fruto de un proyecto de investigación incluido dentro de las líneas de actuación del grupo PAI HUM 556 "Mundialización e Identidad”, de la Universidad de Huelva, así como de las actividades de apoyo a la enseñanza que se vienen haciendo desde la ONG Proyecto Unidad: Seminario Permanente de Solidaridad de la Universidad de Huelva.

Un proyecto de estas características (que se lleva implementando desde hace seis años), ha necesitado del diseño de dispositivos analíticos para la recogida de datos documentales y un estudio de campo diario, con una continuidad a lo largo de todos los cursos académicos mencionados. Para ello, se ha articulado un doble enfoque en la investigación, tanto cuantitativo, como cualitativo. El primero, evidenciado en un estudio de la conflictividad del alumnado y las causas subyacentes que provocan la tensión, tanto en su dimensión ambiental, como humana o normativa, realizado mediante estudios sistemáticos con carácter mensual, analizando variables como las horas, las asignaturas, las asignaciones de grupo, la disposición del aula, las relaciones con sus iguales y el profesorado, la capacidad empática y un largo etcétera de ítems.

En cuanto al enfoque cualitativo, mucho más interesante en el sentir antropológico, ha venido de la mano de las entrevistas semiestructuradas con la población objeto de estudio, tanto alumnado como profesorado y familias, así como de la observación participante en la que, debido a la posición ocupada, constituimos una parte más del proceso, convirtiéndonos en observadoresobservados (Carloni, 2004).

En nuestra intervención se implementan las cuatro componentes metodológicas consideradas en el proyecto: a) el análisis inicial de los datos disponibles sobre la conflictividad en el centro y sus causas explicativas; b) la selección de ítems de análisis lo suficientemente representativos como para aportarnos una visión compleja de la problemática; c) la selección de alumnado en relación a determinados perfiles susceptibles de ser analizados y que pudieran ser extrapolables al resto de la comunidad educativa; y d) el diseño de un programa de intervención con el alumnado en riesgo de exclusión y su concreción en el taller de cocina multicultural.

El plan de trabajo simultanea diferentes actuaciones, manteniendo en la actualidad esa misma dinámica, pues las condiciones de cambio de la sobremodernidad en la que vivimos generan la necesidad de estar continuamente en un proceso de renovación pedagógica y de criterios pues, 
como hemos apuntado con anterioridad, concebimos una educación para el sujeto (Morin, 2004) y no puramente programática.

La primera fase en el desarrollo del proyecto se centra en la recogida de información de todas las variables explicativas de las tramas de conflictividad dentro y fuera del aula, ya que la existencia de conexiones ocultas (Capra, 2003) da lugar a barreras invisibles, pero insalvables en la acción educativa, si no son reconocidas en su contexto. Con carácter mensual se procede a un análisis de los datos obtenidos en la unidad de guardia por medio de un cuestionario que incluye ítems como la hora, el alumno, el profesor, la causa, etc, a fin de determinar pautas de comportamiento entre el alumnado y profesorado, en un intento de desentrañar las barreras ocultas a las que hemos aludido.

La segunda fase se centra en la selección de alumnado y familiares para la implementación del proyecto, creando un perfil antropológico del alumnado potencialmente beneficiario del programa, atendiendo a criterios tales como sus habilidades sociales, su autoestima, el contexto social, las calificaciones o las relaciones del Yo en el contexto comunitario.

La última fase consiste en la puesta en práctica de los talleres de cocina multicultural, diseñados y gestionados por miembros de la comunidad educativa. En esos momentos es donde la observación participante, así como otros sistemas de recogida de información, como las entrevistas semiestructuradas o las actividades y reflexiones realizadas en el marco del taller, cobran vida y se transforman en informaciones privilegiadas de primer orden.

\section{Nuestro banco de pruebas. E1 IES La Marisma y la barriada Diego Sayago}

El I.E.S. La Marisma se ubica en la periferia de dos barriadas onubenses, "El Torrejón” y "Santa Marta". Atiende tres etapas educativas: Educación Secundaria Obligatoria, dos modalidades de bachillerato y dos familias profesionales. Esto hace que el alumnado del centro tenga un perfil social, familiar y educativo muy dispar.

La barriada del Torrejón presenta las características típicas de una zona considerada "en exclusión": altas tasas de desempleo, carencia de infraestructuras, abundancia de infraviviendas y casi ausencia de servicios. En este contexto, buena parte del alumnado que cursa ESO desarrolla un quehacer diario entre problemáticas sociales y personales de importancia, lo cual repercute de manera negativa tanto en su rendimiento académico (presenta un elevado desfase curricular así como de absentismo en clase), como personal (violencia, ausencia de conductas empáticas, escasa asertividad).

Desde hace ya varios años se ha puesto en marcha un proyecto denominado PROYECTO DISTRITO $\mathrm{V}$ que pretende una formación sólida en valores y en el ámbito profesional entre la población de esta barriada. Partiendo de sus peculiaridades (50\% de población gitana y $25 \%$ de población inmigrante con graves problemas de integración social), se pretende partir de un elemento en principio diferenciador, como es el multiculturalismo, para convertirlo, al contrario de lo que pudiera suponerse, en nexo intercultural.

\section{La recogida de información. Instrumentos y tiempos}

Para la selección e identificación de necesidades se tomará una doble vía de trabajo, tanto cuantitativa como cualitativa. Las entrevistas semiestructuradas, así como los estudios de caso conductuales del alumnado y las primeras impresiones obtenidas del modelo de predicción de conductas conflictivas, nos van a aportar un panorama general de la cuestión. De forma corolaria se tomarán los siguientes aspectos en consideración. 


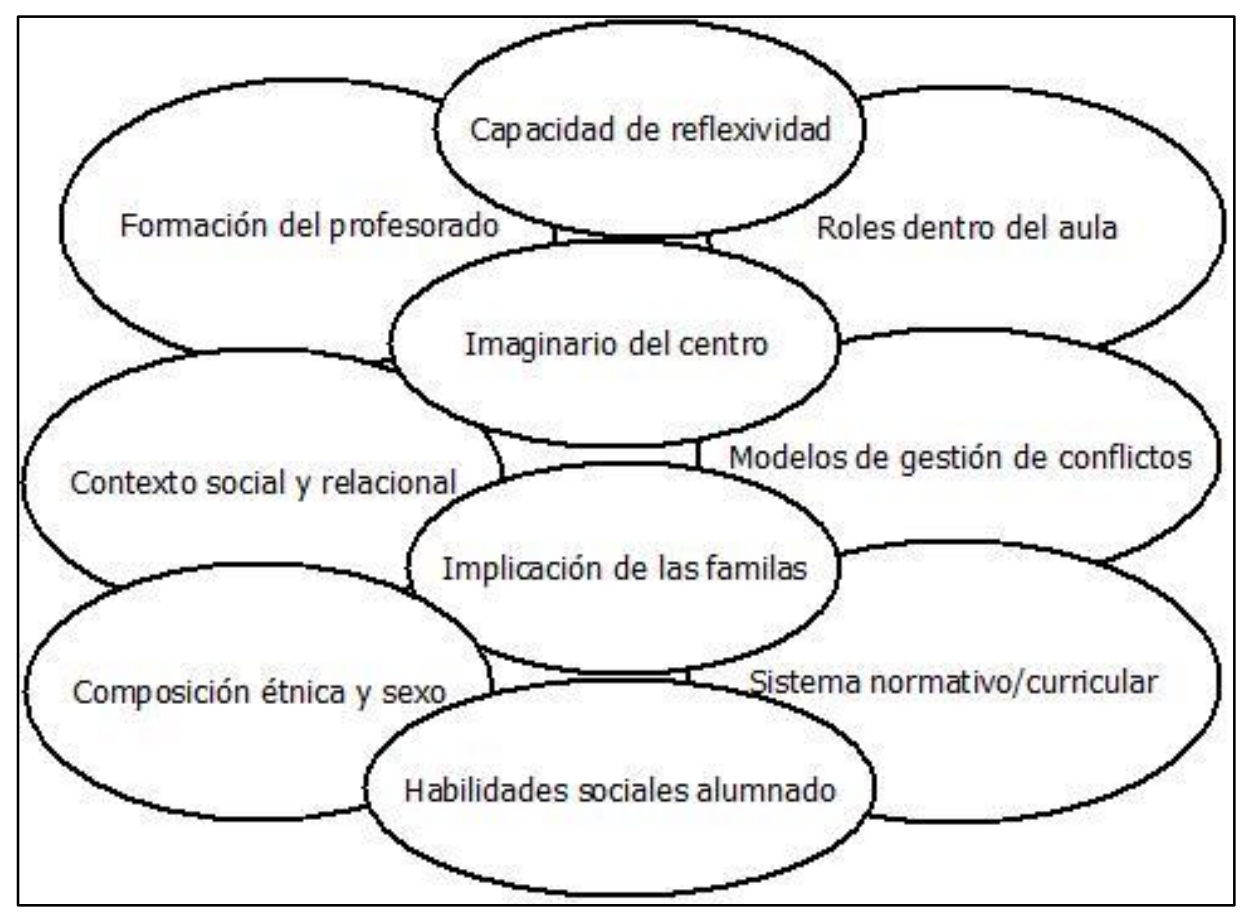

Figura 1. Aspectos que se tomarán en consideración en la investigación.

Las entrevistas se realizarán a lo largo de todo el proceso de investigación, ya que al cambiar cada año de alumnado, varían no sólo los informantes, sino también los contextos particulares en los que se desarrollan como sujetos activos (Pozzoli, 2006), permitiendo adaptar gracias a este medio, las metodologías subyacentes así como el enfoque final del programa. Con una media de 15 entrevistas por cada curso académico, la muestra, tanto en cantidad como calidad, así como su significatividad, va a ser muy productiva en lo referido a las observaciones realizadas, permitiendo incluso un análisis de los cambios comportamentales en el tiempo.

Las entrevistas, siempre semiestructuradas, pues creemos es la que más aporta desde el subconsciente y el entramado simbólico del entrevistado, se dirigen tanto a alumnado como a familias, siempre en contextos donde la acción común es el mundo de la cocina, de ahí que se establezca un vínculo entre la acción y el participante (Hammersley y Atkinson, 2005).

\section{El análisis de datos. La cocina va cobrando forma}

El análisis cruzado de información que se viene produciendo de forma constante por los canales establecidos, permitirá un proceso de categorización dinámico y progresivo, en el que los actores cobran un rol u otro dependiendo del contexto y las variables educativas que fijemos. Al fin y al cabo, la adopción de un modelo analítico basado en el paradigma de la complejidad (Morin, 2004), implica concebir al objeto de estudio en un constante cambio, más aún cuando tratamos con poblaciones que viven en dinámicas donde el caos, el azar o la posibilidad, tres de los principios elementales del pensamiento complejo, están presentes en su cotidianeidad.

Mediante software especializado en análisis de contenidos, así como por medio de simples bases de datos como Excel, se procede a un análisis de todas las variables detectadas a lo largo del proceso de recogida de información, permitiendo categorizar necesidades que dan sentido a toda la actuación. Así, se detectan cinco necesidades perentorias de intervención: 
- Necesidad de cambios en el contexto normativo y de concreción curricular para una mejor adaptación a la realidad de centro.

- Necesidad de cambios en el modelo de intervención en el aula, abandonando parámetros educativos anacrónicos con la situación del alumnado.

- Necesidad de potenciación de las familias en la acción educativa, mediante su incorporación activa en el proceso de enseñanza/aprendizaje, así como mediante un cambio de la relaciones entre todos los miembros de la comunidad educativa.

- Necesidad de un proceso de formación del profesorado en materia de atención a la diversidad.

- Necesidad de una potenciación de las habilidades sociales entre el alumnado, así como en la relación con el profesorado, sus familias y resto de la comunidad educativa.

Esta disimetría de resultados, sin embargo, responderá a una misma dialógica, la presencia de una docencia definida por identificarse con un sistema pedagógico anclado en los parámetros del mecanicismo-reduccionista-lineal-cartesiano, frente a la intención de fomentar la independencia de los sujetos como tales. La solución a todo ello vendrá de la mano de un cambio de la filosofía pedagógica del centro, así como en la formación del profesorado, dotándoles de una alta cualificación para la intervención dirigida al alumnado en riesgo de exclusión.

Sin embargo, aún quedará lo más importante, el aprovechamiento del conflicto como medio de reconducción de las conductas, el verdadero objetivo del proyecto

\section{Resultado. Educando desde los sabores}

Los cambios en los procesos normativos y de intervención en el aula son dos de los principales problemas detectados en el desarrollo de la investigación. El disponer de una normativa en constante cambio, con modificaciones parciales a cada instante, genera una situación de pérdida de rumbo por parte del profesorado, el cual muchas veces no dispone de tiempo para adaptarse a un modelo cuando las autoridades educativas van a volver a cambiarlo. Este fenómeno, a pesar de no ser un problema exclusivo del centro, sino una lacra común en todo el sistema educativo (LOGSE, LOE, LOMCE...), genera muchos problemas de adaptación del sistema, ya que el contexto tan delicado en el que se mueve el centro, con amplias bolsas de marginalidad dentro y fuera, le va a dar una mayor inseguridad. Para combatir este problema, se establecen pautas de control por medio del Proyecto de Centro, dirigidas a unificar finalidades, objetivos, así como metodologías y formas de intervención en el aula, otorgando un espacio de unidad imprescindible.

Con respecto a la necesidad de un proceso de formación del profesorado, especialmente el que imparte los niveles de Secundaria, se recurre a la puesta en marcha de grupos de innovación tales como los de "neurociencia aplicada a la educación", o los de "diseño de proyectos curriculares integrados basados en la complejidad", que pueden ser efectivos para disminuir los déficits observados.

Sin embargo, la gran asignatura pendiente vendrá de la mano de la necesidad de potenciación de las habilidades sociales entre el alumnado. Los datos obtenidos en los informes de derivaciones al aula de guardia por conductas inapropiadas o circunstancias de conflicto grave, indican la existencia de casi un $70 \%$ de alumnado en situación de peligro. Siempre se observa una misma pauta y perfil de alumnado: los eventos se producen principalmente en la hora anterior y posterior a la sesión de recreo, protagonizados por alumnado con graves carencias cognitivas y ausencia de habilidades sociales básicas, tales como la empatía o la escucha. El conflicto, muchas veces buscado expresamente, parece responder a la necesidad de autoafirmación frente al grupo, asumiendo un rol violento para esconder graves carencias (Bordieu, 1999) 
Como consecuencia de esta dinámica, los desfases educativos se van acrecentando hasta hacerlos insalvables, abriéndose aún más la brecha de la exclusión social y la automarginalidad. El sujeto es fagocitado por la imagen de grupo que desea crear, muchas veces rompiendo las reglas como medio de aceptación de sus iguales (Scarduelli, 2005).

Esta forma de poder, en la que el conflicto se utiliza como arma arrojadiza entre sujeto y comunidad, trae consigo graves consecuencias para el alumnado así como para el proceso educativo, pues puede ser considerado como fallido al no lograr su primera finalidad, la inserción como ciudadanos libres de toda la colectividad de estudiantes.

Para corregir esta tendencia, se han implementado diversas experiencias, siendo el "Taller de cocina intercultural" el que arroje mejores dividendos. De esta forma, se parte de una filosofía de fondo, el respeto al sujeto y el desarrollo del mismo a partir del principio de reflexividad (Pozzoli, 2006), en la que el alumno va tomando conciencia de su Yo así como de su pertenencia en comunidad.

El perfil disruptivo del alumnado, la composición étnica multicultural con predominio del colectivo gitano, la existencia de un machismo cultural propio en la comunidad (Téllez, 2013), así como la importancia que se concede a las relaciones de poder mediante la violencia, son puntos de partida a tener en cuenta, pues definen algunas de las variables consideradas en el estudio.

Para poder alcanzar los objetivos previstos, la metodología que se emplea es en todo momento abierta y participativa. En ella, el profesor actúa como guía de las actividades, jugando el rol fundamental el alumnado y las familias voluntarias, compartiéndose el resultado final siempre y cuando se respeten las normas establecidas en asamblea por todos los participantes. Así, por medio de sesiones temáticas, se analizan las problemáticas concretas observadas en el aula a lo largo de la semana, buscándose soluciones. Así, por medio de la reflexividad, el alumnado puede avanzar en la producción de un cambio interior en el que vaya tomando conciencia como ser de su papel entre sus iguales y el resto de la comunidad, evitando la acumulación de más conflicto interno (Pozzoli, 2006)

Una vez resueltas las dudas y consensuadas las estrategias de resolución y prevención de situaciones problemáticas, se pasa a una segunda fase en la que, de forma colaborativa, cada participante asume un rol dentro del proceso del taller gastronómico, encargándose unos del control de la receta y las cantidades, otros de la preelaboración, otros de la elaboración y cocinado y los últimos de los emplatados, todo ello con el trabajo cooperativo de las familias, que se insertarán en el proceso educativo en la medida de sus posibilidades.

Pero para que el taller pueda funcionar, necesita contar con un proceso de preparación previa, en el que intervienen todos los agentes que participan en la acción. De esta forma, en los tiempos previos, se consensua con el alumnado las preparaciones que se deben llevar a cabo, las cuáles son trabajadas desde las competencias digitales disponibles en el centro. En su cuaderno destinado a tal fin, no sólo exponen las cantidades y modos de elaboración sino que, de igual modo, buscan información acerca de propiedades de los alimentos o rasgos culturales a ellos asociados. Con posterioridad y con ayuda del profesor, acuden a supermercados de la zona y realizan la compra de los ingredientes, atendiendo a un triple criterio: la existencia de remanentes en el almacén del centro, las propiedades buscadas en los alimentos y el ajuste a una cantidad de presupuesto concreta. Ello no hace sino generar ciudadanía responsable así como fomentar la autoestima y la autonomía del alumnado.

Paralelamente se contacta con las familias y asociaciones, especialmente la Unión Romaní, para acordar los términos de la actuación en la cocina, sirviendo de colaboradores, ya que la toma de decisiones irá rotando entre el alumnado de forma semanal, de tal suerte que se fomente el espíritu de trabajo de equipo. 


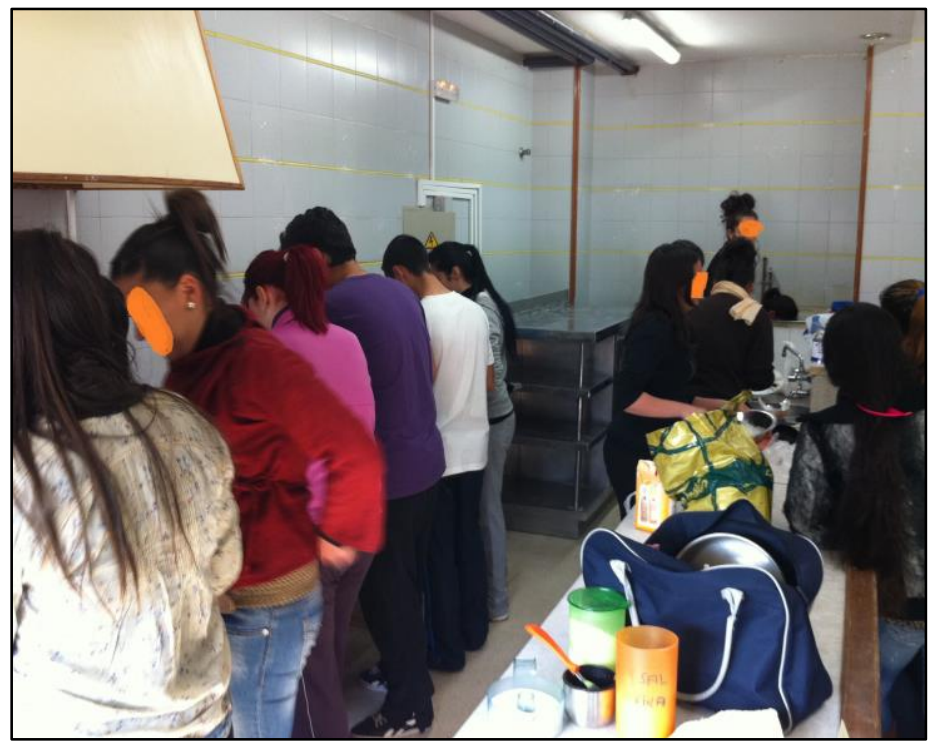

Figura 2. Momento de pre-elaboración en una actividad del taller conductual.

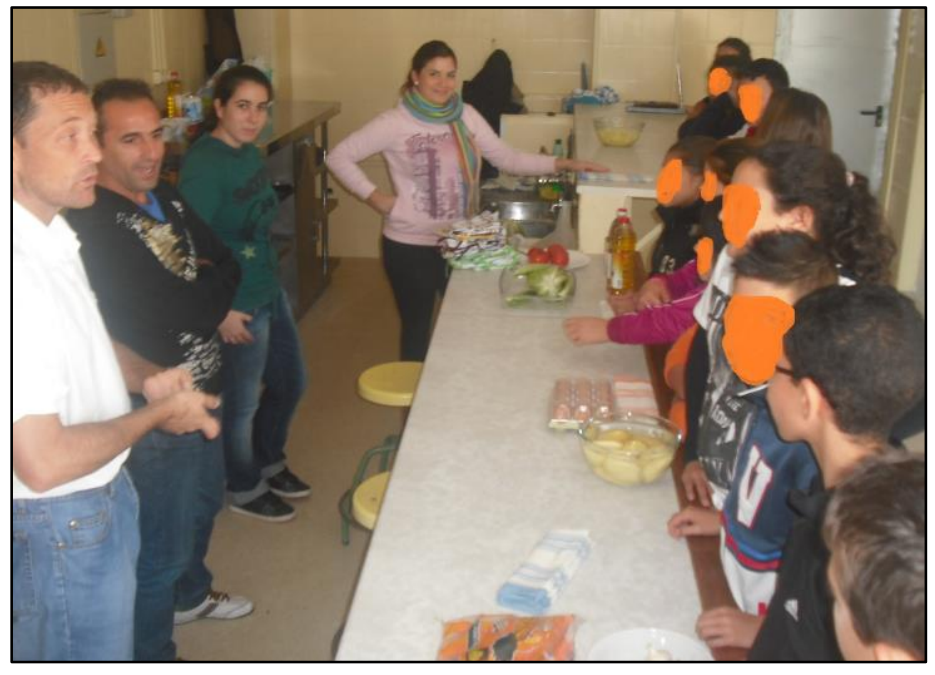

Figura 3. Explicación inicial de la actividad con familia y voluntariado.

El trabajo cooperativo, la creación de unas normas consensuadas de grupo, la eliminación de arquetipos sexuales que se desarrollan cotidianamente en contextos de aula (Téllez, 2012), la importancia de la solidaridad como grupo al haber una interdependencia, así como la generación de un espacio de con-ciencia (del Toro, 2012), donde por medio de la reflexividad se permitirá un crecimiento autónomo del sujeto, hará de este espacio una oportunidad única para el aprendizaje, ya que se rompen las barreras entre los miembros allí presentes y se fomenta una educación donde la empatía cobra protagonismo.

La creación de vínculos afectivos y nuevas formas de relacionarse se convierten en el taller en algo cotidiano. Por medio de la observación participante, hemos podido comprobar cómo hay profesorado que se siente incluso desorientado por el cambio de actitud del alumnado que está desarrollando la actividad, expresando su incredulidad o incluso, en ocasiones, su incomodidad, al no entender dicho proceso de cambio. 
Este fenómeno se produce también entre el alumnado, tanto respecto a sus profesores como a sus familiares e iguales. Verse interactuando en condiciones normalizadas, donde el conflicto se utiliza como fórmula constructiva y no como vía de escape a la violencia, hace que se genere en ellos un sentimiento contrastado, pues contienen en sí una dialógica a la que no están acostumbrados, aprendiendo a convivir con ella en vez de rechazar una de sus partes. Así, no es difícil encontrar entre las notas del cuaderno de campo reflexiones como "si yo antes me llevaba mal con ésta (compañera), ¿cómo es que aquí nos ayudamos y nos llevamos bien?”, siendo esta apreciación más común de lo que en principio pudiera pensarse.

Los resultados de la investigación y la implementación del proyecto se han dejado sentir en el seno de la comunidad educativa. Así, junto a otros programas de intervención con alumnado en riesgo de exclusión, se ha logrado una gradual disminución de la conflictividad en el aula, alcanzándose niveles similares a los de cualquier centro educativo normalizado, lo cual resulta poco menos que increíble teniendo en cuenta el perfil con el que se interviene cotidianamente.

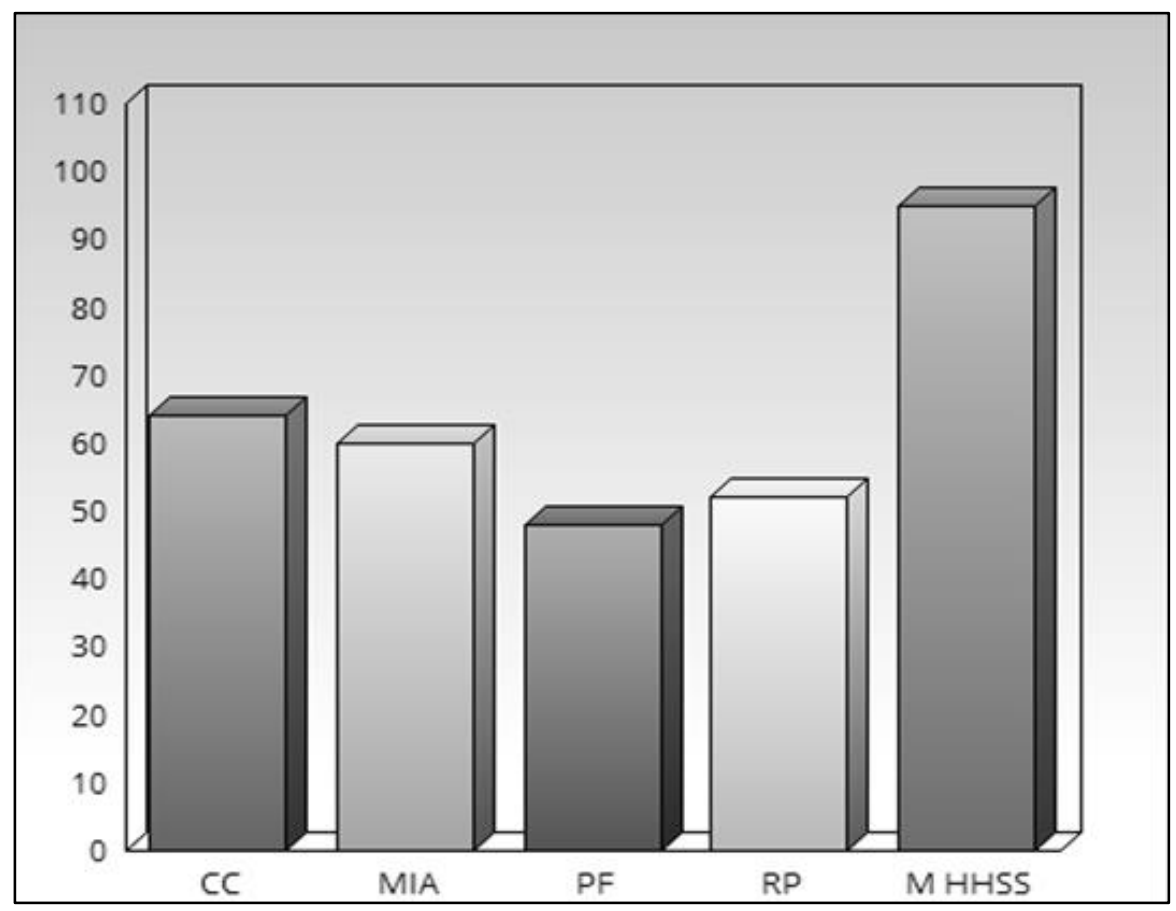

Gráfico 1. Frecuencia de categorías de necesidades detectadas. CC. Contexto normativo y curricular. MIA. Modelos de acción en el aula. PF. Potenciación del rol familiar. RP. Reciclaje profesorado. MHHSS. Mejora habilidades sociales

\section{¿Y todo esto para qué? Una discusión antropoeducativa}

A lo largo de esta investigación, tanto en la fase de recolección de datos como en la puesta en marcha de un sistema de corrección de conductas conflictivas, hemos podido comprobar cómo el fenómeno de vulnerabilidad no se puede explicar sino asumiendo la complejidad del fenómeno así como del individuo.

El llevar seis años ininterrumpidos tomando registros, así como la asunción de una metodología mixta (cuantitativa y cualitativa) a partir de un modelo etnográfico, ha permitido una 
visión muy completa de este fenómeno, pudiendo asignar a cada actor el rol que juega, así como las modificaciones que serían estimables llevar a cabo para una resolución del problema.

En lo referido al profesorado, la falta de una formación específica para su actuación en colectivos de riesgo, ha sido un lastre en los primeros años, aprendiéndose por medio del ensayoerror con la consiguiente pérdida de efectividad de algunas actuaciones. Dicha falta de formación se ha amortiguado mediante una concienzuda labor de cambio de enfoque metodológico basado en la neurociencia, así como por medio de un diseño curricular colaborativo más ajustado a las necesidades del alumnado, quedando aún parcelas pendientes como la intervención directa o las formas de resolución pacífica del conflicto ante determinadas situaciones.

Con respecto a la propia finalidad educativa, se ha abandonado o se ha avanzado en el proceso de abandono de una concepción academicista lineal, propia del pensamiento positivista, para enfocar el proceso en un modelo educativo Botton Up, donde se parte de las necesidades y aspiraciones del alumnado, contemplado como sujeto provisto de reflexividad que, por medio de su autoconocimiento, es capaz de modificarse y generar cambios en el entorno próximo a partir de un proceso de asimilación de sus contradicciones.

El taller de cocina no hace sino potenciar la autonomía de un alumnado constreñido por normas e imposiciones que le asfixia como persona. El disponer de situaciones libres de la coactiva normativa oficial permite generar espacios de con-ciencia a partir de la crítica interior de cada uno de los participantes, lo que contribuye a la formación de personas más libres, activas y con capacidad de decisión, condición indispensable para la superación progresiva de la exclusión social.

\section{Referencias}

Achilli, E. (1996). Práctica docente y diversidad socio-cultural. Rosario: Homo Sapiens.

Aguirre, A. (1995). Etnografía. Metodología cualitativa en la investigación sociocultural. Barcelona: Marcombo. Augé, M (2008). Los no lugares. Espacios del anonimato. Barcelona: Gedisa.

Bolívar, A. y López, L. (2009). Las grandes cifras del fracaso y los riesgos de exclusión educativa. Profesorado. Revista de Currículum y Formación del Profesorado, 13 (3), 50-78.

Bourdieu, P. (1999). La miseria del mundo. México: FCE

Capra, F. (2003). Las conexiones ocultas. Barcelona: Anagrama.

Carabaña, J. (2004). Ni tan grande, ni tan grave ni tan fácil de arreglar. Datos y razones sobre el fracaso escolar. Cuadernos de Información Económica, 180, 131- 140.

Carloni, A. (2004). La lección aplicada del observado observado: curación externa, curación interna. En Actas del VI Congreso de Antropología Aplica y del Simposio: El Sentido Práctico de la Antropología (pp. 110-125). Granada: S.P. Universidad de Granada.

Del Toro, J.M. (2012). Educar con "co-razón”. Bilbao: Desclee de Brouwer.

Escudero, J. (2009). Buenas prácticas y programas extraordinarios de atención al alumnado en riesgo de exclusión educativa. Profesorado. Revista de Currículum y Formación del Profesorado, 13 (3), 107-143.

García, I. y Olmos, A. (2012). Segregaciones y construcción de la diferencia en la escuela. Madrid: Trotta.

Goetz, J.P. y Lecompte, M. D. (1988). Etnografía y diseño cualitativo en investigación educativa. Madrid: Morata.

Hammersley, M. y Atkinson, P. (2005). Etnografía. Métodos de investigación. Barcelona: Paidós.

Jociles, M.I. y Franze, A. (2008). ¿Es la escuela el problema? Madrid: Trotta

Jociles, M.I., Franze, A. y Póveda, D. (2011). Etnografías de la infancia y de la adolescencia. Madrid: Catarata 
Luengo, J. (2004). Paradigmas de gobernación y de exclusión en la educación. México: Pomares.

Malinowski, B. (1995). Los argonautas del Pacifico Occidental. Barcelona: Península.

Morín, E. (2004). Introducción al pensamiento complejo. México: Gedisa.

Pozzoli, M.T. (2006). El sujeto de la complejidad. La construcción de un Modelo Teórico Transdisciplinar (eco-psico-socio-histórico-educativo). Polis, Revista de la Universidad Bolivariana, 5 (15), 1-14.

Scarduelli, P. (2005). Per un'antropologia del XXI secolo. Tribalismo urbano e consumo dell'esotico. Roma: Squilibri.

Serra, C. (2004). Etnografía escolar, etnografía de la educación. Revista de Educación, 334, 165-176.

Soriano, E. (2009). Vivir entre culturas: una nueva sociedad. Madrid: La Muralla.

Soriano, E. (2011). El valor de la educación en un mundo globalizado. Madrid: La Muralla

Subirats, J. (2004). Pobreza y exclusión social. Un análisis de la realidad Española y europea. Barcelona: Fundación la Caixa.

Téllez, A. (2013). El análisis de la adolescencia desde la Antropología y la perspectiva de género. Interacçoes, 25, 52-73

Vélaz, C. (2005). Cómo prevenir el rechazo y la exclusión social. Cuadernos de Pedagogía, 348, 58-61.

Woods, P. (1987). La escuela por dentro. La etnografía en la investigación educativa. Barcelona: Paidós-MEC.

\section{Sobre el autor}

Autor: Juan Carlos Romero Villadóniga

Institución: Grupo HUM 556 de la UHU

E-mail: jucarovi66@gmail.com

Información biográfica: Máster en Patrimonio Histórico y Natural por la UHU, Máster en Antropología Aplicada por la UCLM, Doctor en Patrimonio Histórico por la UHU, así como miembro del GRUPO PAI HUM 556 Mundialización e Identidad de la UHU. Alterna su faceta investigadora con la docencia en centros de difícil desempeño desde el año 1998. Coordina proyectos de Ayuda al Desarrollo en contextos desfavorecidos de España y Argentina por medio de la ONG Proyecto Unidad: Seminario Permanente de Solidaridad de la UHU. En su dimensión como investigador coordina un proyecto sobre la influencia de la importancia de las vulnerabilidades sociales en contextos educativos, así como asesora en calidad de antropólogo, en un programa de recuperación de la memoria oral y el patrimonio intangible en poblaciones marismeñas onubenses. Autor de dos libros sobre conformación de la identidad y 20 artículos, algunos de ellos en prensa.

ORCID: orcid.org/0000-0002-0835-1636 


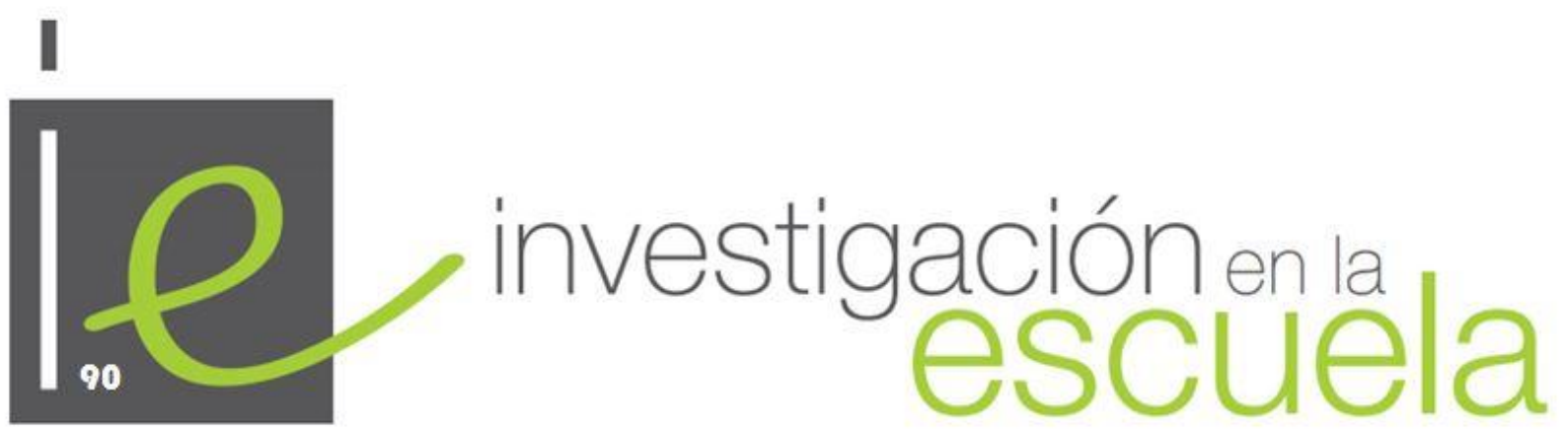

Revista académica evaluada por pares y de acceso abierto

Número 90

28 de diciembre de 2016

ISSN 2443-991

(c)

SOMERIGHISRESERVED Los/as lectores/as pueden copiar, mostrar, y distribuir este artículo, siempre y cuando se de crédito y atribución al autor/es y a Investigación en la Escuela, se distribuya con propósitos no-comerciales, no se altere o transforme el trabajo original. Más detalles de la licencia de Creative Commons se encuentran en http://creativecommons.org/licenses/by-nc-sa/3.0 Cualquier otro uso debe ser aprobado en conjunto por el autor/es, o Investigación en la Escuela.

Contribuya con comentarios y sugerencias en la web de la revista. Por errores y sugerencias contacte a investigacionescuela@,ddcc.uhu.es 
Investigación en la escuela

Consejo de dirección: Ana Rivero García (Universidad de Sevilla), Nicolás de Alba Fernández (Universidad de Sevilla), Pedro Cañal de León (Universidad de Sevilla), Francisco F. García Pérez (Universidad de Sevilla), Gabriel Travé González, (Universidad de Huelva), Francisco F. Pozuelos Estrada (Universidad de Huelva)

Dirección: Ana Rivero García y Nicolás de Alba Fernández

Técnico de edición: Francisco Javier López Sánchez

\section{Consejo editorial}

José Félix Angulo Rasco. Universidad de Cádiz Rosa Ma Ávila Ruiz. Universidad de Sevilla Pilar Azcárate Goded. Universidad de Cádiz Juan Bautista Martínez Rodríguez. Universidad de Granada

Nieves Blanco García. Universidad de Málaga Fernando Barragán Medero. Universidad de La Laguna José Carrillo Yáñez. Universidad de Huelva José Contreras Domingo. Universidad de Barcelona. Luis C. Contreras González. Universidad de Huelva Ana $\mathbf{M}^{\mathbf{a}}$ Criado García-Legaz. Universidad de Sevilla Rosario Cubero Pérez. Universidad de Sevilla José $\mathbf{M}^{\mathbf{a}}$ Cuenca López. Universidad de Huelva Jesús Estepa Giménez. Universidad de Huelva Rafael Feito Alonso. Universidad Complutense (Madrid)

Francisco José García Gallardo. Universidad de Huelva

Soledad García Gómez. Universidad de Sevilla J. Eduardo García Díaz. Universidad de Sevilla
Fernando Hernández Hernández. Universidad de Barcelona

Salvador Llinares Ciscar. Universidad de Alicante Alfonso Luque Lozano. Universidad de Sevilla Rosa Martín del Pozo. Universidad Complutense (Madrid)

José Martín Toscano. IES Fernando Herrera (Sevilla) Jaume Martínez Bonafé. Universidad de Valencia F. Javier Merchán Iglesias. Universidad de Sevilla Emilia Moreno Sánchez. Universidad de Huelva. Rosario Ortega Ruiz. Universidad de Córdoba Antonio de Pro Bueno. Universidad de Murcia Fco. de Paula Rodríguez Miranda. Universidad de Huelva

Pedro Sáenz-López Buñuel. Universidad de Huelva Antoni Santisteban Fernández. Universidad Autónoma (Barcelona)

Emilio Solís Ramírez. Catedrático de IES. $\mathbf{M}^{\mathbf{a}}$ Victoria Sánchez García. Universidad de Sevilla. Magdalena Suárez Ortega. Universidad de Sevilla

\section{Consejo asesor}

Manuel Area Moreira. Universidad de La Laguna

Jaume Carbonell. Director Cuadernos de Pedagogía. Barcelona

César Coll. Universidad de Barcelona

Christopher Day. Universidad de Nothingham. U.K.

Juan Delval. Universidad Nacional de Educación a Distancia

John Elliott. Universidad de East Anglia. Norwich. U.K.

José Gimeno Sacritán. Universidad de Valencia

André Giordan. Universidad de Paris VII y Ginebra

Francisco Imbernón. Universidad de Barcelona

Ángel Pérez Gómez. Universidad de Málaga

Rafael Porlán Ariza. Universidad de Sevilla

Francesco Tonucci. Instituto de Pedagogía del C.N.R. Roma

Jurjo Torres Santomé. Universidad de A Coruña 
